\title{
Effect of a Plume Reduction in Segmented Electrode Hall Thruster
}

\author{
Y. Raitses, L.A. Dorf, A A. Litvak and N.J. Fisch \\ Princeton Plasma Physics Laboratory, P.O. Box 451, \\ Princeton University, Princeton NJ 08540
}

\begin{abstract}
A segmented electrode, which is placed at the thruster exit, is shown to affect thruster operation in several ways, whether the electrode produce low emission current or no emission current, although there appear to be advantages to the more emissive segmented electrode. Measured by plume divergence, the performance of Hall thruster operation, even with only one power supply, can approach or surpass that of non segmented operation over a range of parameter regimes, including the low gas rate regime. This allows the flexibility in operation of segmented electrode thrusters in variable thrust regimes.
\end{abstract}




\section{Introduction}

The main advantage of using electric propulsion for spacecraft orbit control is larger jet velocities $(10-100 \mathrm{~km} / \mathrm{s})$, which enables significant savings in the propellant mass as compared to chemical rockets $(<3 \mathrm{~km} / \mathrm{s}$ ). The Hall thruster is particularly suited for many space applications, which are limited in time and fuel mass. The principle of Hall thruster operation is based on electrostatic acceleration of ions in crossed electric and magnetic fields applied in a quasi-neutral plasma of a dc electric discharge. ${ }^{1}$ Hall thrusters are typically coaxial (See Fig. 1), consisting of a magnetic circuit, anode, which can also a gas distributor, ceramic channel and cathode neutralizer. A gas propellant, which is typically Xenon, enters the channel through the anode and is ionized by impact with energetic electrons of the electrical discharge. The applied radial magnetic field impedes the axial electron motion towards the anode. The impeded electrons can then more effectively ionize the propellant atoms and support a significant axial electric field with equipotentials along the magnetic field lines $\left(\mathrm{E}=-\mathrm{v}_{\mathrm{e}} \times \mathrm{B}\right)$. The axial field accelerates the ions towards the channel exhaust, where they neutralized by electrons from the cathodeneutralizer. The thrust is a reaction force to this electrostatic acceleration, applied to the magnetic circuit.

Existing Hall thrusters can efficiently produce large jet velocities, $10-20 \mathrm{~km} / \mathrm{s}$, in the input power range from several hundreds watts to a few tens of kilowatt. ${ }^{2}$ Since ions acceleration takes place in a quasi-neutral plasma these thrusters are not a subject for space charge limitation that allows to achieve higher current and thrust densities than conventional electrostatic ion thrusters at discharge voltages of hundreds volts. In 
addition to this particular advantage, Hall thrusters are griddles and therefore may be also attractive for many industrial applications as plasma sources.

The key drawback of Hall thrusters as compared to ion thrusters is large beam divergence, which affect significant wall losses inside the channel $(\sim 20 \%$ of the input power $\left.{ }^{3}\right)$ and large plume angle $\left(90^{0}\right.$ of plume angle $\left.{ }^{4}\right)$. In a number of references different mechanisms, which could potentially affect this beam divergence were suggested and analyzed $^{5,6,7}$. For example, because of a high electron temperature $(10-20 \mathrm{eV})$, equipotentials can deviate from the concave magnetic field surfaces affecting defocusing electric field in the channel. ${ }^{5}$ On the other hand, the presence curved outward magnetic field surfaces at the exit of the channel and as well as a fringing magnetic field outside the thruster can also affect defocusing of the beam. ${ }^{6}$ In addition, non uniform distribution of the ion production in the accelerating region can lead to a spread of ion energy distribution and, as a result, to the presence of slow ions affected more strongly by defocusing electric fields ${ }^{7}$.

In the existing Hall thrusters, the electric field distribution is controlled mainly by the magnetic field profile along the thruster channel, which is $\partial \mathrm{B}_{\mathrm{r}} / \partial \mathrm{z}>0 .{ }^{1}$ However, implementation of this control by the axial gradient is limited by design and magnetic properties of the magnetic circuit. $^{2}$ A Hall thruster with segmented emissive electrodes along the thruster channel can enable an additional control of the electric field distribution and therefore may have certain advantages over conventional designs. ${ }^{8,9}$ Dielectric insulators separate the electrodes, with segments held at different potentials through separate power supplies. Electrons, entering the channel, flow through the electrodes. The radial magnetic field provides magnetic insulation so that very abrupt 
potential drops, and a very localized acceleration region, can be established in the thruster channel. The localization can be in a region of concave magnetic field for maximum focusing, resulting in less plume divergence.

Flexibly designed laboratory prototype segmented electrode Hall thrusters were built and deployed in the Princeton Plasma Physics Laboratory (PPPL). The segmented electrodes are annular rings, placed either inside the outer ceramic vessel wall or over the inner vessel wall. This is in order to prevent breakdowns between biased segmented electrodes and their shortening by sputtering products during the thruster start and operation. As a result, the same magnetic field lines crossing a conductive surface of an electrode, for example, at the inner wall, and a dielectric surface at the opposite outer wall. It turns out that such an introduction of segmented electrodes has a telling effect on the thruster operation in ways not anticipated by any simple theory. Not only is their precise placement along the channel significant, but so is their precise shape and emissivity. Moreover, even low emissive segments held at floating potential can affect thruster operation significantly. ${ }^{9}$

In the present work, we describe results of more comprehensive experiments at more operating conditions and with a low emissive segmented electrode, which was placed at three different locations along the region with curved outward magnetic field lines at the thruster exit. It can be expected that in such configurations, the thruster may represents some combination between the anode layer thruster with metal walls ${ }^{2}$ and Hall (so called magnetic layer) thruster with dielectric walls. In addition to propulsion applications, there is some basic interest in studying of physics of Hall acceleration regime when the 
magnetic field lines connect channel walls made from two different materials with different electrical and secondary emission properties.

\section{Experimental Setup}

The vacuum system consists of a $28 \mathrm{~m}^{3}$ stainless steel vacuum vessel of a diameter $2.29 \mathrm{~m}$ and length $8.38 \mathrm{~m}$ equipped with a 35 " diffusion pump and mechanical vacuum roots pumping system. At a xenon gas flow rate of about $23 \mathrm{SCCM}$, the measured background pressure was about 24 microtorr, corresponding to a pumping speed somewhat larger than 12,000 1/s. To prevent contamination of the thruster and diagnostics due to backstreaming during warm up and cooling of the diffusion pump, this pump is connected to the vessel through a 35 " right angle valve. In addition, the thruster and most of the diagnostics are located at the end of the large vacuum vessel, opposite to the right angle flange, which also reduces backstreaming contamination.

Two commercial (Tylan) gas flow controllers FC-260 (0-50 SCCM and 0-10 SCCM) were used in order to control and measure a Xenon gas flow to the anode and cathode of the thruster. In addition to the Millipore LR -250 readout and control box, a pressure gauge was used to verify calibration, stability and repeatability of the flow meters in the laboratory environment.

A set of commercial voltage and current regulated power supplies was used in order to support ignition and operation of the thruster and as well as operation of probe diagnostics. The main discharge was supported by a $1 \mathrm{kV}, 10 \mathrm{~A}$ switching power supply. Separate voltage regulated and current regulated supplies were used for biasing of the segmented electrode and electromagnetic coils, respectively. 
The1 kW laboratory Hall thruster (See Fig. 2) has modular design to facilitate studies of the thruster operation under different configurations, with and without segmented electrodes. The outer diameter of the channel, which is characteristic channel dimension, is $90 \mathrm{~mm}$. The magnetic field distribution in the thruster channel was numerically simulated by using a commercial electromagnetic software package (See Fig. 3) and measured by a gauss meter. As an example, the magnetic field profile (at a coil current of 2 A) along the thruster axis near the median of the thruster channel is shown in Fig. 4.

The segmented electrode, which is refered below as the negative side or NS electrode, is placed along the channel axis on low potential side at the inner wall. In the present set of experiments, the electrode has about $1 \mathrm{~mm}$ thickness of $\mathrm{LaB}_{6}$, which was plated in a rhenium mesh to allow a strong structure of the emissive layer. This mesh was mounted on a molybdenum substrate ring of $3 \mathrm{~mm}$ for each electrode. The length of the NS electrodes was $4 \mathrm{~mm}$. Fig. 5 shows this segmented electrode attached to inner wall of the thruster channel. The voltage potential of the segmented electrode can be floating or biased from separate power supply or from the main discharge power supply.

The thruster is suspended on an arm of high resolution $(0.5 \mathrm{mN})$ pendulum-type thrust stand. ${ }^{9}$ The total ion current of the emerging plasma jet and plume parameters (mean cosine angle and plume angle for $90 \%$ of the flux) were measured with a flat electrostatic Langmuir probe. The probe, which is a circular disk of about $2 \mathrm{~cm}$ diameter made from a low sputtering material, was mounted on a positioning mechanism. This mechanism enables the probe to move on a circle with the center on the thruster axis, while the radius of the circle can be varied from $33 \mathrm{~cm}$ to about $80 \mathrm{~cm}$. The voltage drop 
due to the probe current was measured on $100 \Omega$ low inductance shunt. Probe position was measured by a rotating potentiometer. Control of the probe motion and probe measurements were performed by using a data acquisition system which includes an A-TE series board of National Instruments and Labview software. Fig. 6 shows the thruster suspended on the thrust stand and the probe setup.

PC based digital scopes and spectrum analyzers (DAQ 5102) were used to monitor and characterize discharge oscillations. In voltage mode, the discharge current oscillations were measured on a low impedance shunt.

Operating temperature of the inner segmented electrode was measured by a thermocouple, which was fixed on a molybdenum substrate, while surface temperature of LaB6 was obtained by an infrared imaging camera TH5104, observing the electrode through a $\mathrm{ZnSn}$ window. The camera was calibrated in a set of experiments with LaB6 samples heated to $600^{\circ} \mathrm{C}$.

\section{Experimental Procedure}

The thruster operation was investigated in 8 configurations, including without segmented electrodes (referred to as WS). The segmented configurations are as follows: with one negatively biased segment positioned at the edge of the inner magnetic pole (NS1), with the negative segment shifted at $2 \mathrm{~mm}$ (NS2) and $4 \mathrm{~mm}$ (NS3) upstream of the thruster channel relative to the edge of the inner magnetic pole. Fig. 4 shows these positions of the segmented electrodes relative to the magnetic field profile. To evaluate the effect of the segmented electrode, a set of experiments was carried out with a ceramic 
extension (CE) of the same size as the NS electrode and placed at the same position as the segmented electrode in the NS1 case.

For each configuration, measurements were performed for an anode Xenon gas flow rate of $1.7,2$ and $2.5 \mathrm{mg} / \mathrm{s}$. In addition, operating regimes with $3 \mathrm{mg} / \mathrm{s}$ of the anode flow rate were also investigated when it would not cause breakdown or unstable operation caused by deposition of the NS electrode material on the outer channel wall.

In order to verify reproducibility of operating regimes, almost each operating point with segmented electrodes was repeated at the beginning, at the middle, and at the end of each thruster run. The discharge current and thrust indicated changes no larger than the measurement error in most of the stable operating points.

The thruster efficiency, $\eta=T^{2} / m P_{e}^{11}$, was deduced from the thrust, $T$, mass flow, $m$ and input power, $P_{e}$, measurements. In all these results, the mass flow rate through the cathode, which was a $0.3 \mathrm{mg} / \mathrm{s}$, was not taken into account. The error in thrust and performance determination is given in Ref. 10. For example, in the measured operating regimes of the thruster, thrust error was taken equal to the thrust resolution, while efficiency error was no more than $\pm 5 \%$ of the measured value.

Using an electrostatic probe placed at a distance of $33 \mathrm{~cm}$ from the thruster exit, the total ion current and plume angle for $90 \%$ of the total ion flux, $I_{i}$, from the thruster were measured. Assuming single ionization of Xenon propellant, the propellant utilization, $I_{i} / I_{m}$, was estimated, where $I_{m}=e M_{i} / m, e, M_{i}$ are the equivalent mass flow current, electron charge and ion mass, respectively. ${ }^{11}$ For certain operating points, plume measurements were repeated several times at certain angles. The repeatability of the full plume angle was about \pm 4 degrees. 


\section{Experimental Results}

During operation with the NS electrode, the maximum electrode temperature was measured by a thermocouple when the thruster was at steady state. In the case of NS1, the surface temperature of LaB6 was also measured with an infrared camera. The maximum temperature from thermocouple (about $1040^{\circ} \mathrm{C}$ ) was obtained when the electrode was at NS1 position, while the thruster operated at discharge voltage of $300 \mathrm{~V}$ and mass flow rate of $3 \mathrm{mg} / \mathrm{s}$. At this operating point, infrared measurements showed the maximum of $1100^{\circ} \mathrm{C}$ at LaB6 surface. The temperature dropped when the input power was reduced. In addition, at the same operating point, the temperature measured by thermocouple was about $900^{\circ} \mathrm{C}$ and $700^{\circ} \mathrm{C}$, for NS2 and NS electrode cases, respectively. The reduction with the power is most probably due to a decrease of energy of ions hitting the wall. An alternative explanation might be that, by shifting the electrode from NS1 to NS3 along the thruster channel, the ion flux to the segmented electrode decreases, also leading to the observed reduction of the electrode temperature.

Illustrative curves of voltage versus current characteristics (V-I) measured at two anode mass flow rates of $1.7 \mathrm{mg} / \mathrm{s}$ and $2.5 \mathrm{mg} / \mathrm{s}$ for 7 different configurations of the thruster: WS, NS1, NS2 and NS3 are shown in Fig. 7. Here, in each segmented electrode case, the V-I curve was measured for the NS electrode under floating potential and cathode bias. Each point on these curves was obtained at the minimum of the discharge current versus the magnetic field ${ }^{12}$. In addition, Fig. 8 shows the propellant utilization versus the discharge voltage for the same configuration at $1.7 \mathrm{mg} / \mathrm{s}$ and $2 \mathrm{mg} / \mathrm{s}$. Figs. 9 
and 10 compare V-I characteristics and propellant utilization the thruster without segmented, with the CE channel and NS1.

As can be seen from V-I curves, at both mass flow rates and at a given discharge voltage, the discharge current measured without segmented electrode was generally less than that with segmented electrodes for a variety of different configurations and operating regimes. As we move the NS electrode upstream in the channel, the V-I characteristics shift towards larger currents at given discharge voltage and mass flow rate. In addition, the current tends to increase at all three locations when the cathode bias was applied to the segmented electrode. However, the discharge current measured at $1.7 \mathrm{mg} / \mathrm{s}$ for the NS3 under floating potential is less than that measured for the WS configuration. Moreover, at $2.5 \mathrm{mg} / \mathrm{s}$, as the gas flow rate is increased, the discharge current, which at the thruster exit is mainly carried by ions, increases too.

At most operating points, the propellant utilization is higher with the "without segment" (WS) configuration than with any negative side (NS) segmented cases for mass flow rates $1.7 \mathrm{mg} / \mathrm{s}$ and $2.0 \mathrm{mg} / \mathrm{s}$ (see Fig. 8). Similar results were measured with anode mass flow rates of $2.5 \mathrm{mg} / \mathrm{s}$ and $3 \mathrm{mg} / \mathrm{s}$. Consistent with lower propellant utilization and larger discharge current, the ratio of the ion current to the discharge current is also larger for the "without segment" (WS) case.

When the ceramic extension (CE) was placed at the position NS1, the discharge current increased even more than it was in the case of the NS1 electrode at that position for the same discharge voltage and mass flow rate. This increase, which was typical at all mass flow rates, is illustrated in Fig. 9. However, the ion propellant utilization (Fig. 10) 
almost did not change and therefore, the ratio of the ion current to the discharge current is less compared to what it is for the WS configuration of the thruster.

The plume angle versus the discharge voltage measured at two mass flow rates 1.7 $\mathrm{mg} / \mathrm{s}$ and $2.5 \mathrm{mg} / \mathrm{s}$ and different configuration of the thruster are shown in Fig. 11. Note that at the small mass flow rate of $1.7 \mathrm{mg} / \mathrm{s}$ rate, a smaller plume half-angle (about $10^{\circ}$ ) occurred under NS configuration, in particular, under the biased NS2 case. When the mass flow rate is increased, the differences become comparable to the error in the reproducibility of the probe measurements. However, there is an indication of generally smaller plumes for the WS case for mass flow rates higher than $2.5 \mathrm{mg} / \mathrm{s}$.

\section{Discussion and Summary}

An explanation of the behavior of the thruster with the NS electrode cases might be along the following lines: According to Refs. 2 and 13, under typical Hall thruster and anode layer thruster conditions, say $\mathrm{Te} \sim 10-30 \mathrm{eV}, \mathrm{Vi} \sim 10^{3} \mathrm{eV}$, the secondary emission, $\delta$, from metal wall of the thruster channel is negligible. As a result, no "cooling" of electrons takes place in the channel with metal walls. In this case, the electrons gain a kinetic energy, which is randomized by collisions and oscillations, on a shorter distance than it is with ceramic walls. This may increase the gradient of electron pressure, both tending to affect the voltage potential distributions and the radial plasma sheaths. For example, a reduction of the acceleration and ionization region and an increase of the discharge current were experimentally observed in ref 14 , in which sputtering of a metal target placed outside the thruster caused metalization of ceramic walls. Here, an increase in the discharge current as compared to the "clean" channel walls was attributed to 
discharge current oscillations. In addition, the anode layer thrusters have also shorter accelerating layer, while with the magnetic field twice stronger than in Hall thrusters with ceramic walls. $^{2}$

The increased values of the discharge current at given mass flow rates, which were observed in the experiments with floating segmented electrodes (See Fig. 7), may result from such a metalization of the outer wall by sputtering of the negative side segmented electrode placed on the inner wall. In order to prevent this contamination, the outer wall of the channel had a set of small grooves, which allowed a stable long duration operation of the thruster and reproducible measurements. Nevertheless, trace amounts of the electrode material, were indeed detected in post-run inspection of the thruster channel walls by X-ray spectrometer. In addition, after long run of the thruster, the presence of a conductive layer was observed even with an ohmmeter. This is, in particular, in the NS1 electrode case, much smaller in the NS2, but not in the NS3.

The observed differences in the channel metalization for the NS thruster cases seems to be obvious result since more energetic ions arrive to the edge of thruster channel ${ }^{7,11}$, which in the present set of experiments corresponded to the location of NS3. As a result, stronger metalization of the outer ceramic walls took place in this electrode case. However, in contrary to ref. 14, amplitude of discharge current oscillations measured in all NS electrode cases were not larger, but smaller than in the WS case. Therefore, a possible cause of the increased discharge current in the floating NS cases is a smaller magnetic insulation of the acceleration region with a larger electric field as compared to the WS case. Indeed, although "optimal" values of the coils current (magnetic field) in the NS cases were larger by roughly $10-30 \%$ than in the WS case, we could not succeed 
to reach twice amplitudes of the optimal magnetic field as it is typical for the anode layer thrusters. This limitation was due to the overheating of the electromagnetic coils and saturation of the magnetic core at large gas flow rates. As an indication of the reduced magnetic insulation, larger fraction of the axial electron current for the NS cases can be derived from results of V-I characteristics and propellant utilization versus the discharge voltage (Figs.7, 8, respectively).

When the segmented electrode is biased under the cathode potential it can collect ions from the plasma and, as a result, to contribute to the discharge current, as an additional electric circuit path parallel to the main circuit between the anode and cathodeneutralizer. This is most probably why the discharge current with the biased segmented electrodes was larger than in the corresponding floating cases.

Fig. 12 shows the current to NS segmented electrode at the cathode bias at 3 different positions: NS1, NS2 and NS3 and at two mass flow rates, $1.7 \mathrm{mg} / \mathrm{s}$ and $2.5 \mathrm{mg} / \mathrm{s}$. As can be seen, when the segmented electrode is moved inside the thruster channel, the current tends to decrease. At a given mass flow rate and electrode position, the measured current to segmented electrode tends also to decrease as the discharge voltage is increased. Note that from the above explanations this current is likely an ion current.

Note that the reduction of the current to the segmented electrode, as it is moved upstream to the maximum magnetic field, does not necessarily mean a reduction of the effect of this electrode on the acceleration and ionization region. As can be seen in Fig. 13, the floating potential of the segmented electrode does not change with the discharge voltage in the NS1 configuration, but it drops with the NS3 case when the discharge voltage is above $250 \mathrm{~V}$ at $1.7 \mathrm{mg} / \mathrm{s}$ and above 220 at $2.5 \mathrm{mg} / \mathrm{s}$. The difference in the 
behavior of the floating potential may be also attributed to changes in the length or motion of the acceleration region as the discharge voltage is varied. In addition, the behavior of the potential with gas flow rate at a given discharge voltage may be also attributed to changes in the acceleration region length as the electron temperature and atomic density increase with the mass flow and more ionization collisions take place closer to the thruster exit.

In the CE case (See Fig. 9), the increase of the discharge current as compared to the WS at given discharge voltage can also be, in principle, attributed to the sputtering of the ceramic extension with following coating of the channel walls by a boron nitride dust. In ref. 14 similar behavior of the discharge current was observed with a quartz dust on a boron nitride channel. However, since for Silicon $\delta \sim 1$ at $300 \mathrm{eV}^{14}$, a quarts dust affects an increase in the electron temperature similar to metal walls. On the other hand, in our experiments, the dust and channel were of the same ceramic material and therefore, had the same secondary emission coefficients. As a possible explanation, in addition to electron wall collisions, wall recombination losses at the ceramic extension may result an increase of the atomic density at the thruster exit. That may be an additional source of a degradation of the magnetic insulation in the acceleration region relevant for the CE case and, at least, for the NS3 segmented electrode.

In principle, depending on their location, either relative to each other or to the magnetic field and the electron temperature, two channel wall segmented electrodes made from different materials should be able to affect defocusing or focusing of the ion beam and as a result the propellant utilization and plume. For example, it was suggested in ref 15 , that due to a larger potential difference between the plasma and the floating metal 
wall the ion flux to the wall will increase in the most of the acceleration and ionization region leading to an increase of ion losses. In addition, ref. 14 attributed the measured reduction of the ion current with metalized channel walls to a degradation of the ionization conditions and an increase of ion energy distribution in a shorted ionization and acceleration region. As a result, it could be expected that the plume angle should be also increase in the channel with metal walls as compared to with ceramic walls. Both these explanations may be relevant for the results of the propellant utilization with the segmented electrode thruster (See Fig. 8).

The observed reduction of the plume angle with segmented electrodes and only slight degradation of the propellant utilization within accuracy of the probe measurements at some discharge voltages (See, for example, NS2 case at $2 \mathrm{mg} / \mathrm{s}$, Fig. 10) and small mass flow rates involves probably different mechanisms. These improvements over the WS case may be attributed, for example, to different secondary emission coefficients of the ceramic and metal walls along the same magnetic field lines, resulting in different sheath and pre sheath potential drops those values and thickness may be affected by the operating conditions and inclination of the magnetic field lines to the electrode surface. ${ }^{16}$ Another possible explanation is that at small mass flow rates the ionization mean free path is increased, leading to less efficient ionization and larger ion losses in conventional thruster configuration. ${ }^{17}$ The metal walls may enable to increase the electron temperature and, as a result, to provide the ion formation in a shorter region with less losses.

Note, as the segmented electrode is biased under the cathode potential, it may also collect slow ions, which contribute to the plume at large angles ${ }^{7}$. 
Figs. 14 and 15 show thrust and thruster efficiency versus the discharge voltage for mass flow rates at $1.7 \mathrm{mg} / \mathrm{s}$ and $2.5 \mathrm{mg} / \mathrm{s}$, for each of 7 configurations with NS segmented electrode and without segmented electrode. Note that the thrust and efficiency are somewhat better with the non-segmented configuration at most of the operating points, voltage and mass flow rates, and different configurations, as might be expected given the measurement of propellant utilization versus the discharge voltage (Fig. 8.) and the V-I characteristics (Fig. 7). Note too that this rather small general decrease in thrust and efficiency for segmented electrode operation comes with the more significant general improvement in plume divergence.

Finally, it has to be mentioned that better understanding of the effect of the segmented electrode on integral characteristics of the Hall thruster and its plume requires local measurements of the plasma parameters inside the thruster and theoretical model.

This work was supported by the U.S. DOE under Contract No. DE-ACO2-76CHO3073.

\section{References}

1. Morozov, A. I., Esipchuk, Yu. V., Kapulkin A. M., Neverovskii, V. A. and Smirnov, V. A., Effect of the magnetic field on a closed electron drift accelerator, Sov. Phys. Tech. Phys., 17, 1972 pp. 482-487.

2. Zhurin, V. V., Kaufman, H. R. and Robinson, R. S., Physics of closed drift thrusters, Plasma Sources Sci. Technol, 8, 1999. 
3. Martinez-Sanchez, M. and Pollard J. E., Spacecraft Electric Propulsion-An Overview, Journal of Propulsion and Power, 5, Sept.-Oct. 1998, pp. 688-699.

4. Askhabov, S. N. et al, Sov. J. Plasma Physics, 7, 125, 1981.

5. Morozov, A., I., Bugrova, A. I., Ermolenko, V. A. and Lein, L. A., A study of ion formation in a Hall accelerator, Sov. Phys. Tech. Phys, 33(2), 1988, pp. 185-187.

6. Keidar M. and Boyd I. D., Effect of a magnetic field on the plasma plume from Hall thrusters, Journal of Applied Physics, 86(9), Nov. 1999.

7. King, L. B. and Gallimore, A. D., Ionic and Neutral Particle Transport Property Measurements in the Plume of an SPT-100, AIAA paper 96-2712, Lake Buena Vista, Fl, July 1996.

8. Fruchtman, A., Fisch, N. J., "Modeling the Hall Thruster," AIAA 98-3500, Cleveland, OH, 1998.

9. Fisch, N.J., Raitses, Y., Litvak, A. A., and Dorf, L. A., "Design and Operation of Hall Thrusters with Segmented Electrodes” AIAA 99-2572, Los Angeles, CA , June 1999.

10. Brophy J. R. Stationary Plasma Thruster Evaluation in Russia, JPL publications, March 1992.

11. Kim, V., Main Physical Features and Processes determining the Performance of SPT, Journal of Propulsion and Power, 14(5), Sept-Oct 1998, pp. 736-743.

12. Raitses, Y., Ashkenazy, J., "Discharge Characteristics of Hall Current Accelerators," Proc. XVII Int. Symp. on Discharges and Electrical Insulation in Vacuum, Berkeley, CA (July, 1996). 
13. Morozov, A. I., Conditions for efficient current transport by near-wall conduction, Sov. Phys. Tech. Phys., 32(8), 1987, pp. 901-904.

14.Bugrova, A. I., Desyatkov, A. V., Morozov, A. I. And Kharchevnikov, Rearrangement of a discharge in a steady-state plasma engine due to the dusting of insulators, Plasma Physics reports, 22(4), 1996, pp. 302-305.

15. Egorov, B. B., Kim, V., Semenov, A. A. and Shkabran, I. I., Near wall processes and their influence on Hall thruster operation, in book "Ion Injectors and Plasma Accelerators,” Ed. By Morozov, A. I. and Semashko, N. N., Publishing house: Energoatomizdat, Moscow, 1990, pp. 55-68. [in Russian].

16. Beilis, I. I. and Keidar, M., Sheath and presheath structure in the plasma-wall transition layer in an oblique magnetic field, Physics of Plasmas, 5(5) May 1998, pp. $1545-1553$.

17. Raitses, Y., Ashkenazy, J and Guelman, M., Propellant utilization in Hall thrusters, Journal of Propulsion and Power, 14(2), 247, March-April 1998. 


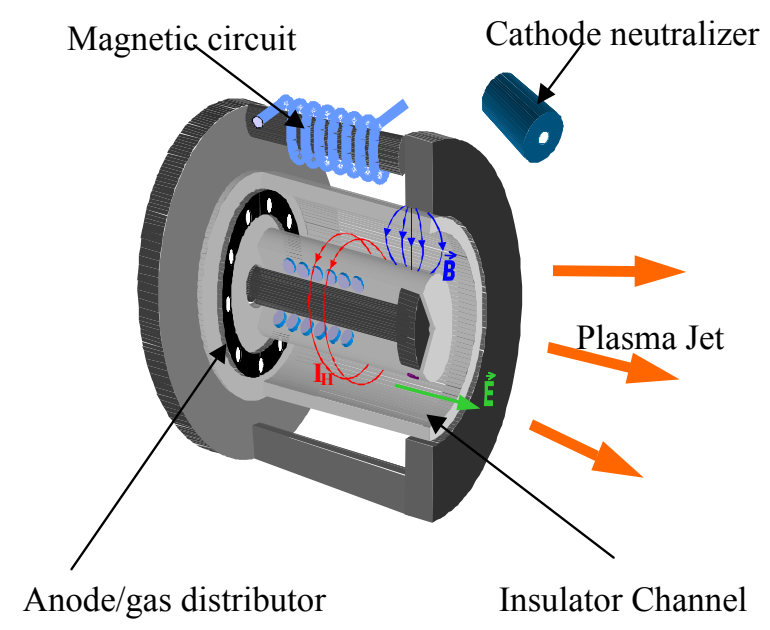

Fig. 1. Schematic drawing of the Hall thruster.

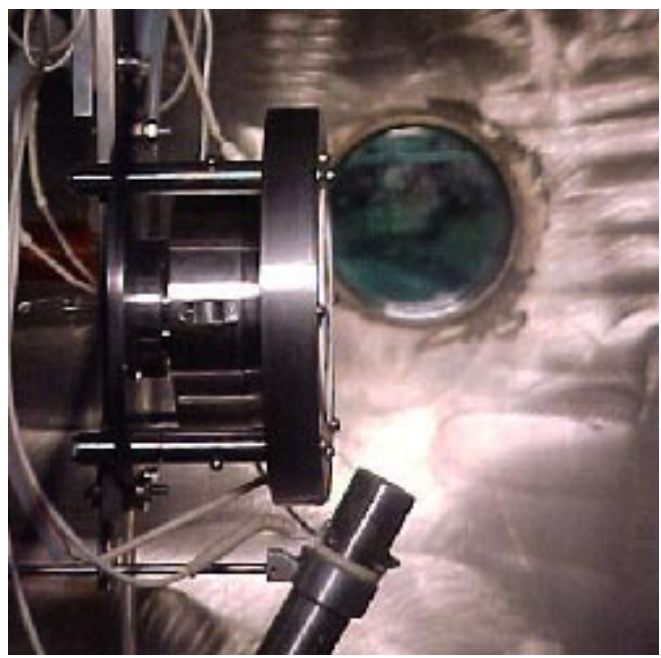

Fig. 2. PPPL laboratory model Hall thruster. 


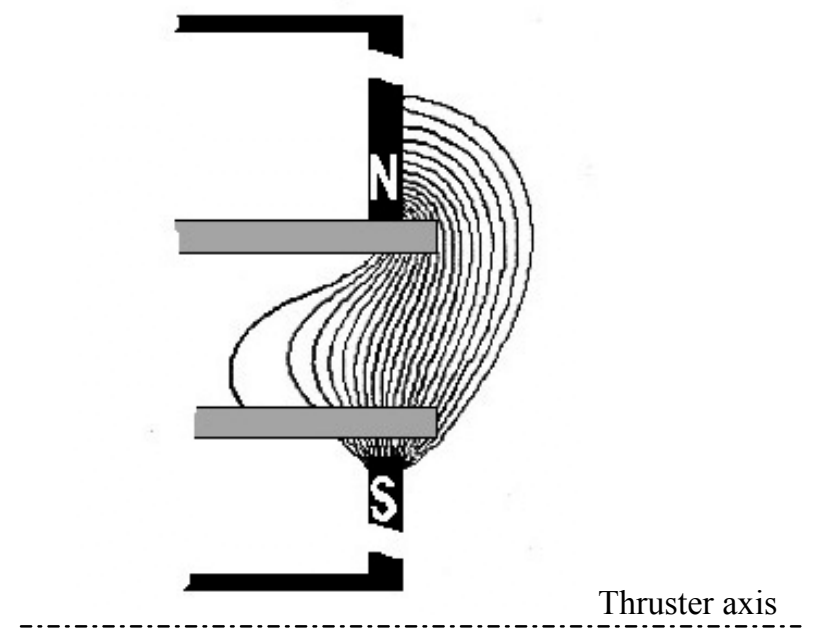

Fig. 3. Simulated Magnetic field.

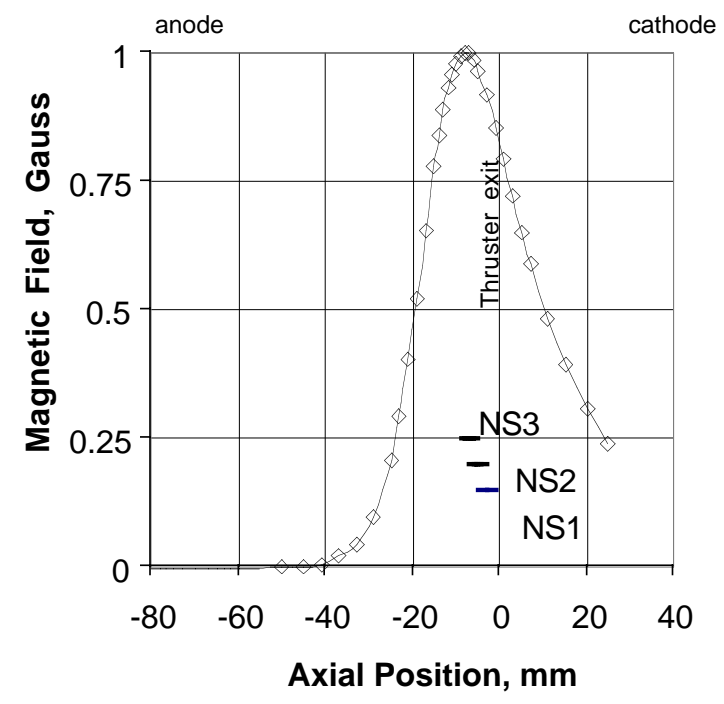

Fig. 4 Measured profile of the radial component of the magnetic field along the thruster axis near the channel median. Magnetic field is produced by a 2.1 A electromagnet current. NS1, NS2, NS3, are locations of inner segmented electrode along thruster axis. 


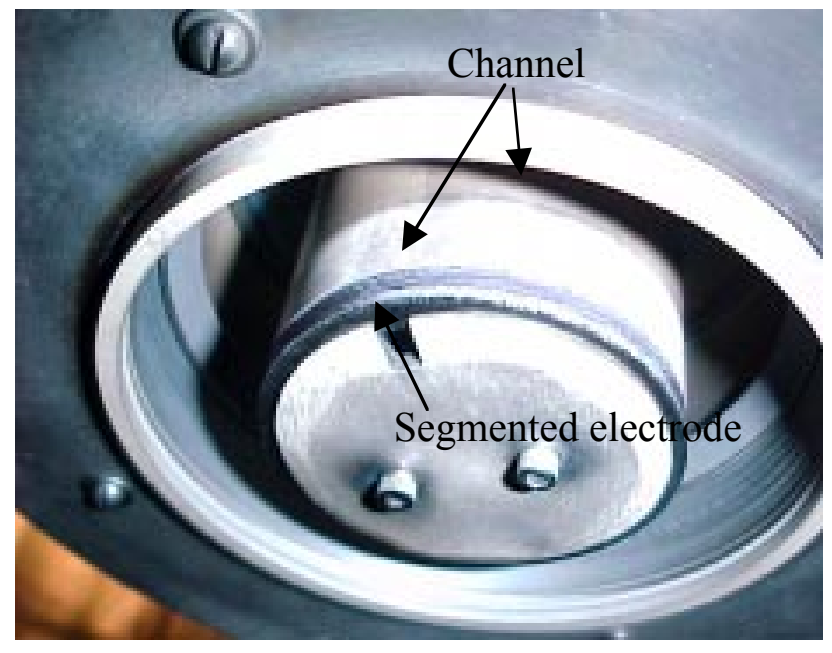

Fig. 5. The negative side (NS) segmented electrode attached to the inner wall of the ceramic channel.

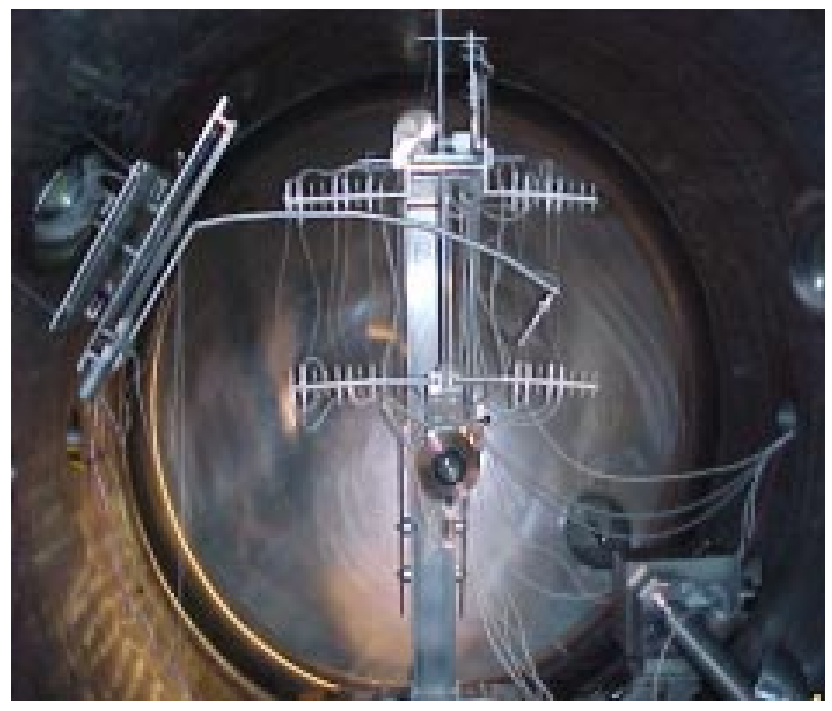

Fig. 6. Thrust stand and probe setup. 
(a)

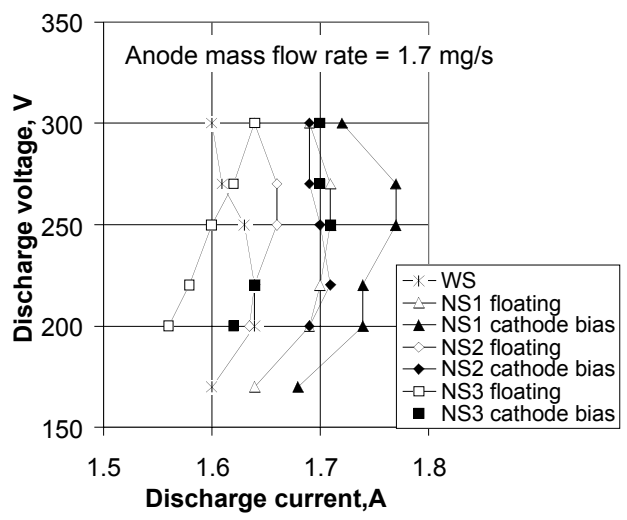

(b)

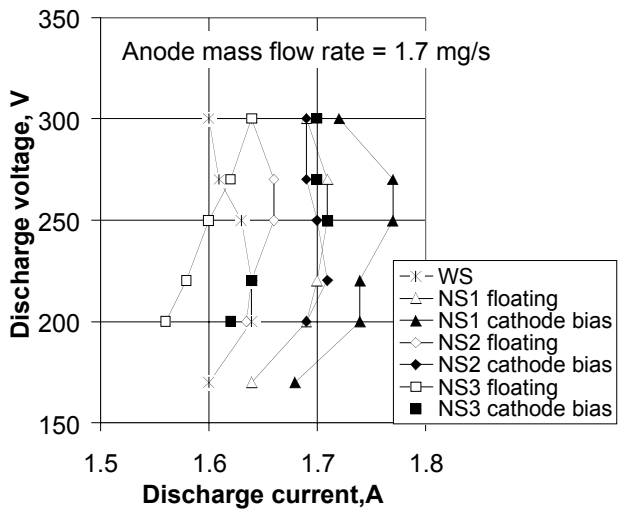

Fig. 7. V-I characteristics of thruster for 7 thruster configurations: WS; and NS1, NS2, NS3 at (a) $1.7 \mathrm{mg} / \mathrm{s}$ and (b) $2.5 \mathrm{mg} / \mathrm{s}$. 
(a)

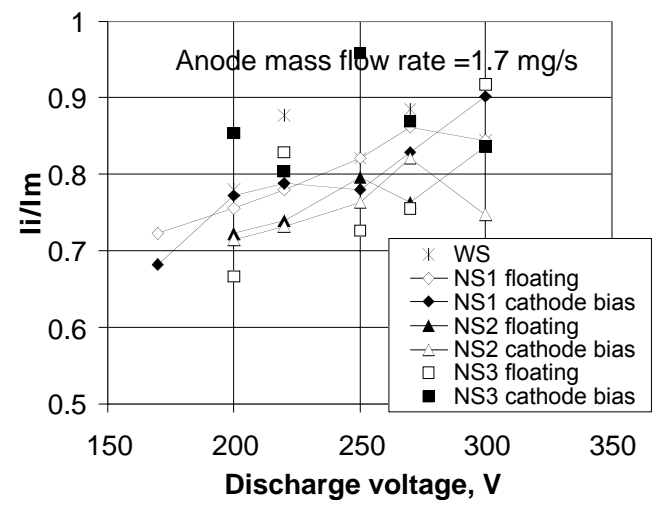

(b)

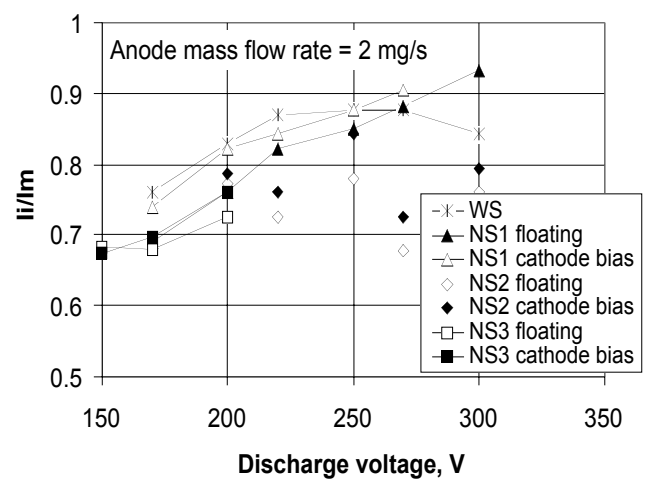

Fig. 8. Propellant utilization versus the discharge voltage measured for 7 thruster configurations WS, NS1, NS2, NS3, at (a) $1.7 \mathrm{mg} / \mathrm{s}$ and (b) $2 \mathrm{mg} / \mathrm{s}$. 


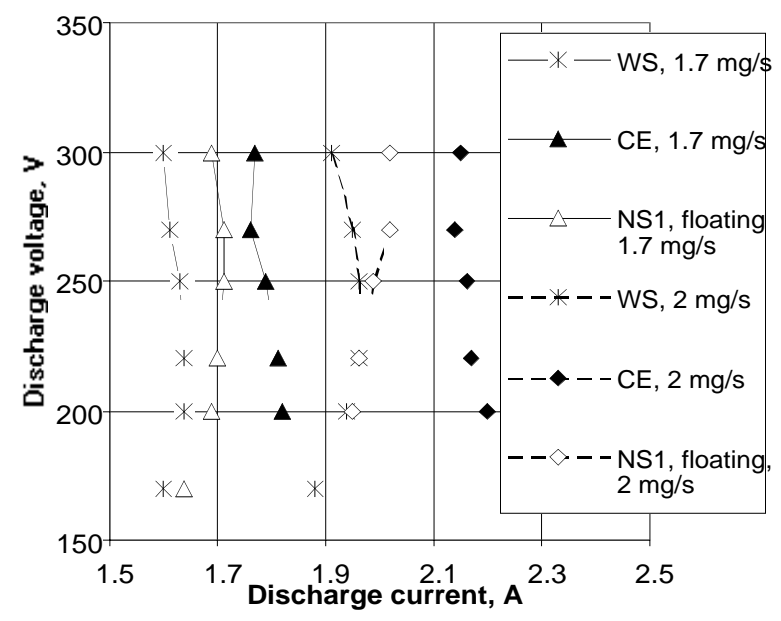

Fig. 9. V-I characteristics for three thruster configurations WS, NS1 (floating and under cathode bias), $\mathrm{CE}$, at (a) $1.7 \mathrm{mg} / \mathrm{s}$ and (b) $2 \mathrm{mg} / \mathrm{s}$.

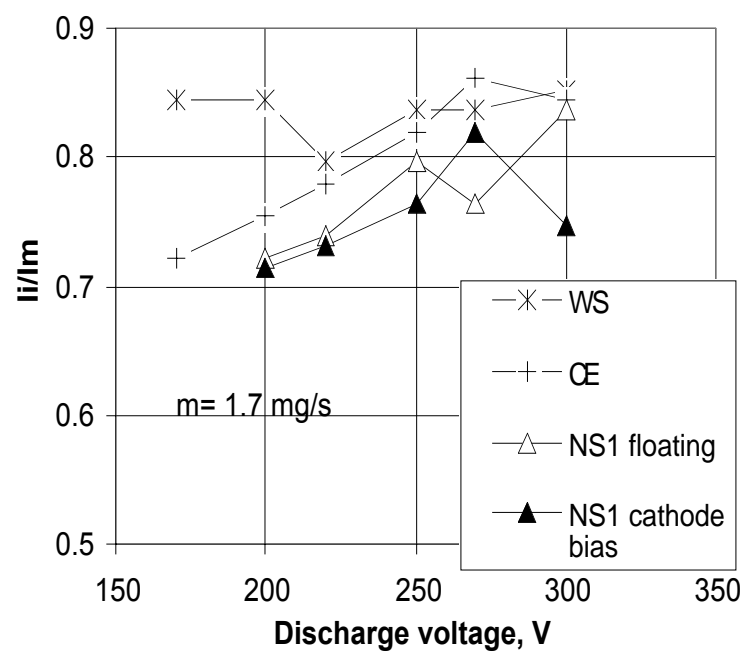

Fig. 10. Propellant utilization versus discharge voltage measured for four thruster configurations WS, NS1, CE, at (a) $1.7 \mathrm{mg} / \mathrm{s}$ and (b) $2 \mathrm{mg} / \mathrm{s}$. 
(a)

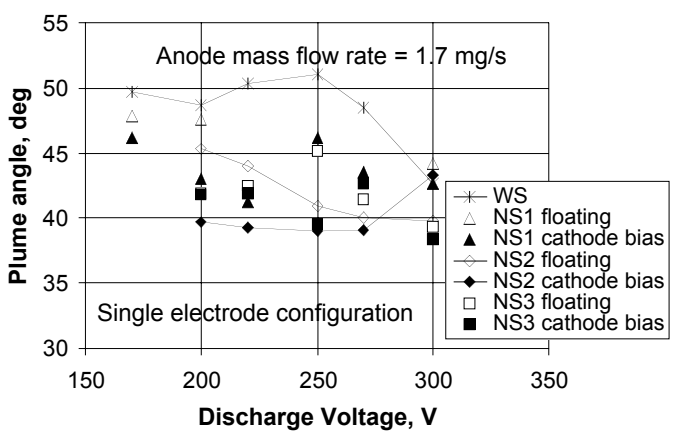

(b)

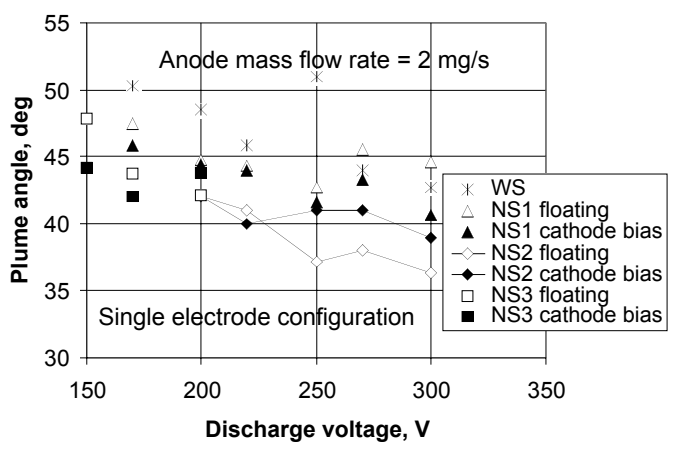

(c)

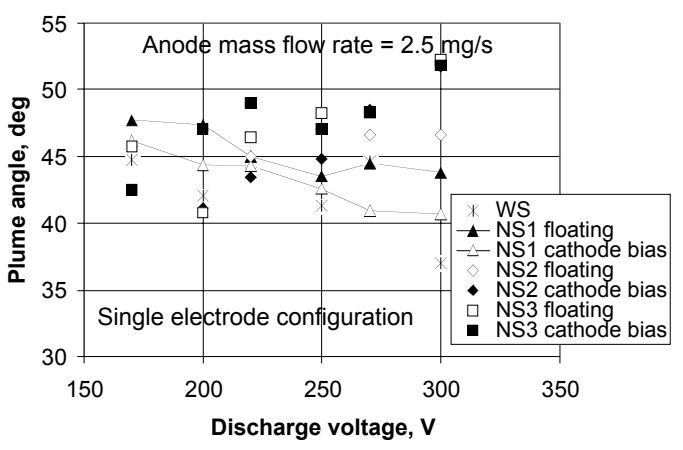

Fig. 11. Plume angle estimated for $90 \%$ of the measured ion flux from the thruster of different configurations (WS,NS1,2 and 3, floating, biased) at 4 different mass flow rates. 


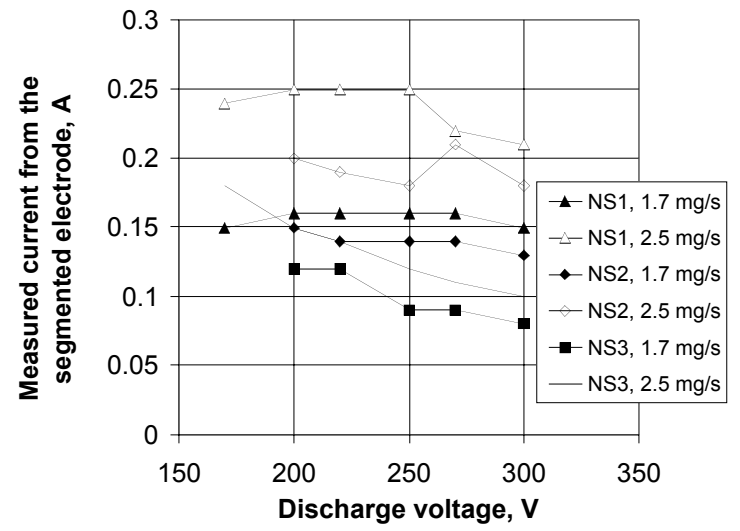

Fig. 12. Current to the segmented electrode, measured for three segmented electrode configurations, NS1, NS2, NS3 measured at two mass flow rates 1.7 and $2.5 \mathrm{mg} / \mathrm{s}$.

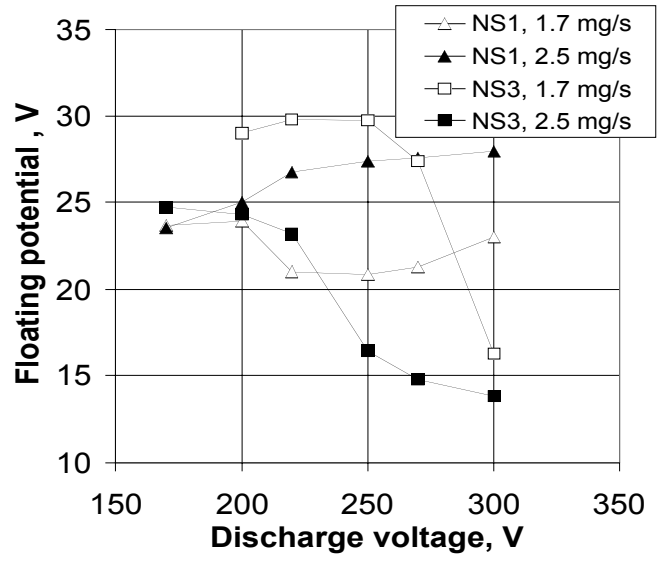

Fig. 13. Floating potential measured between the cathode and segmented electrode for two segmented electrode configurations NS1 and NS3 at two mass flow rates, 1.7 and $2.5 \mathrm{mg} / \mathrm{s}$. 
(a)

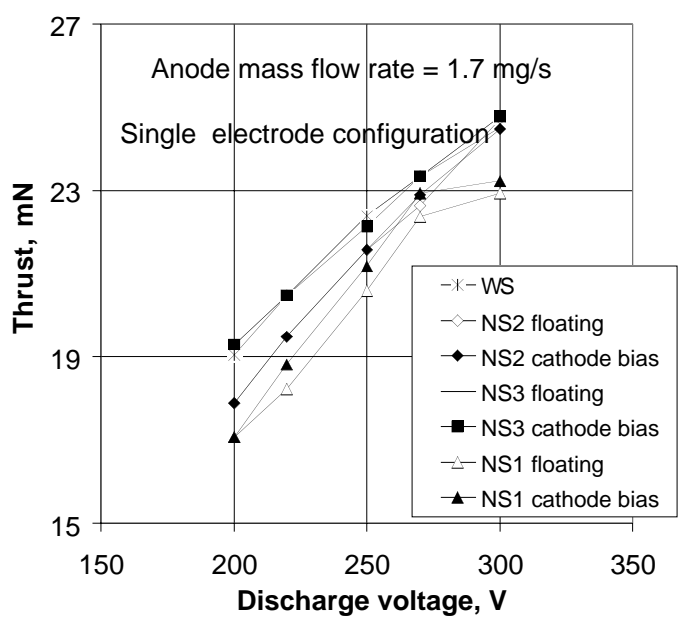

(b)

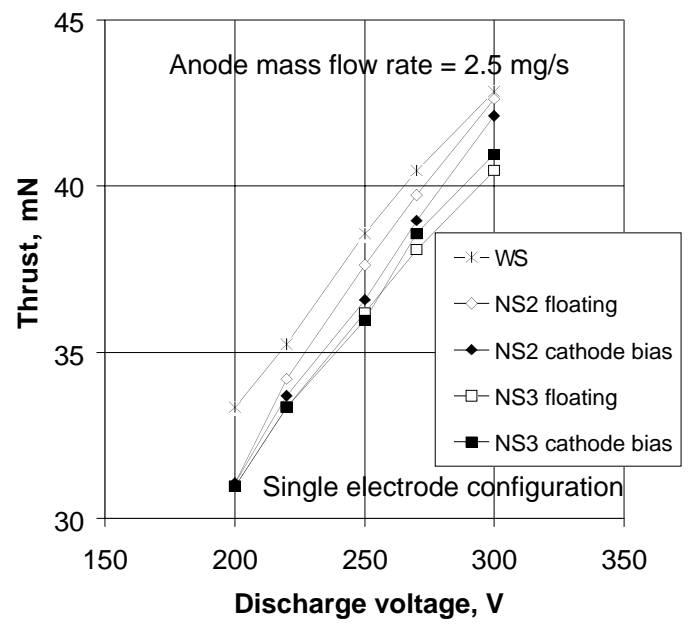

Fig. 14. Thrust versus discharge voltage measured at 2 mass flow rates, (a) $1.7 \mathrm{mg} / \mathrm{s}$ for WS, NS1,2, 3 and (b) $2.5 \mathrm{mg} / \mathrm{s}$ for WS, NS2,3. 
(a)

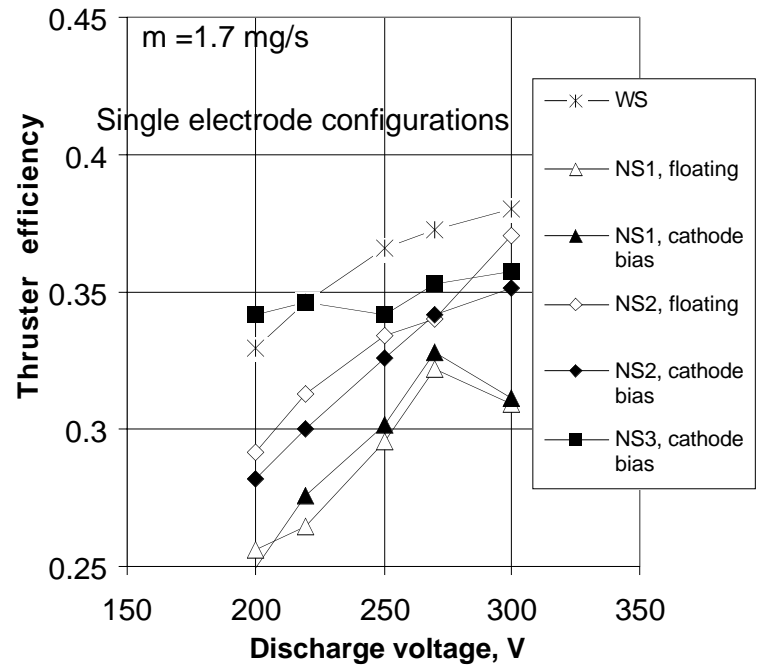

(b)

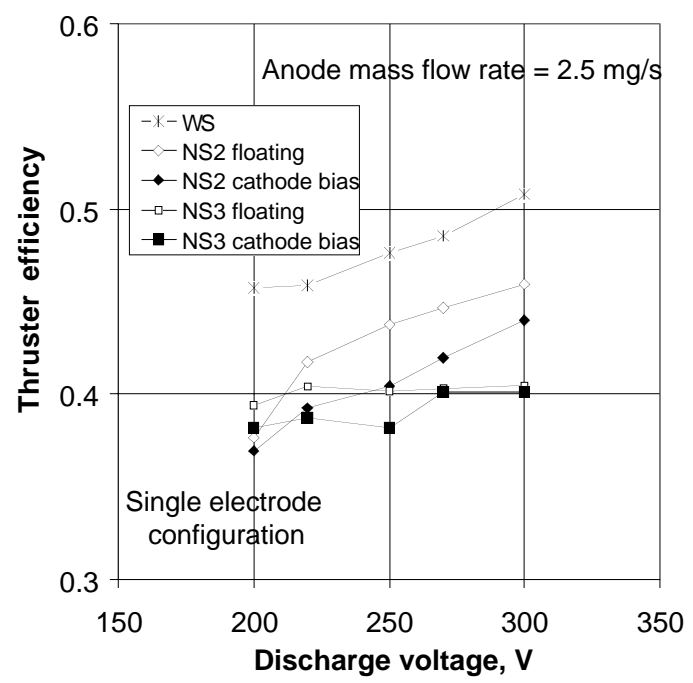

Fig. 15. Thruster efficiency versus discharge voltage measured for WS and NS1, NS2 and NS3 floating and biased configurations at two mass flow rates: (a) $1.7 \mathrm{mg} / \mathrm{s}$ and (b) 2.5 $\mathrm{mg} / \mathrm{s}$. 